\title{
Constrained Multiobjective Equilibrium Optimizer Algorithm for Solving Combined Economic Emission Dispatch Problem
}

\author{
M. A. El-Shorbagy $\mathbb{D}^{1,2}$ and A. A. Mousa $\mathbb{i D}^{3}$ \\ ${ }^{1}$ Department of Mathematics, College of Science and Humanities in Al-Kharj, Prince Sattam Bin Abdulaziz University, \\ Al-Kharj 11942, Saudi Arabia \\ ${ }^{2}$ Department of Basic Engineering Science, Faculty of Engineering, Menoufia University, Shebin El-Kom 32511, Egypt \\ ${ }^{3}$ Department of Mathematics and Statistics, College of Science, Taif University, P.O. Box 11099, Taif 21944, Saudi Arabia \\ Correspondence should be addressed to M. A. El-Shorbagy; mohammed_shorbagy@yahoo.com
}

Received 14 November 2020; Revised 22 December 2020; Accepted 29 December 2020; Published 15 January 2021

Academic Editor: Baogui Xin

Copyright (c) 2021 M. A. El-Shorbagy and A. A. Mousa. This is an open access article distributed under the Creative Commons Attribution License, which permits unrestricted use, distribution, and reproduction in any medium, provided the original work is properly cited.

\begin{abstract}
This research implements a recent evolutionary-based algorithm of equilibrium optimizer to resolve the constrained combined economic emission dispatch problem. This problem has two objective functions that represent the minimizing of generation costs and minimizing the emission of environmental pollution caused by generators. The proposed algorithm integrates the dominant criteria for multiobjective functions that allow the decision-maker to detect all the Pareto boundaries of constrained combined economic emission dispatch problem. In order to save the effort for the decision-maker to select the best compromise alternative, a cluster study was carried out to minimize the size of the Pareto boundary to an acceptable size, representing all the characteristics of the main Pareto frontier. On the other hand, in order to deal with the infringement of constraints, a repair algorithm was used to preserve the viability of the particles. The proposed algorithm is applied to solve the standard 30-bus IEEE system with 6 generators to validate its robustness and efficiency to produce a well-distributed Pareto frontier for constrained combined economic emission dispatch problem. Compared with other studies, good results in solving constrained combined economic emission dispatch problem are obtained and a reasonable reduced Pareto set is found.
\end{abstract}

\section{Introduction}

The robust and efficient economic planning, operation, and distribution of power systems have always presided a vital role in the power system industry. Saving a small percent in the operation of the power systems produces a reasonable reduction in the operating cost and in the amount of fuel consumed [1]. Finding the optimum operating cost is the key purpose of the classic constrained combined economic emission dispatch problem (CEEDP) [2]. Recently, for large scale electric power system, modern system optimization theory methods are applied with the cost savings [3]. There are three directions to solve CEEDP [4].

Traditionally, the first direction is to simplify the multiobjective optimization problem (MOP) to a single objective problem. Traditional methods used to convert MOP into a single objective problem are either the aggregating of objective functions as in the weighted sum method or the optimization of the most important objective and the treatment of others as constraints as in the $\varepsilon$-constraint method or the penalty factor approach [5]. Then, various numerical optimization methods have been employed to handle this single objective problem such as the augmented Lagrangian method and gradient method, for example, in weighted sum [6], the $\varepsilon$-constraint method [7]. The most important weaknesses of these methods are that it cannot deal with nonconvex function and tends to find weak set of nondominated solutions. On the other hand, goal programming is also implemented to deal with CEEDP [8]. In this approach, a specified target is assigned for each objective to be achieved and then aims to minimize the deviation from the desired targets to the objective functions. 
The second direction is to solve the single objective CEEDP by any single objective meta-heuristics algorithm such as artificial bee colony (ABC) [9], gravitational search algorithm (GSA) [10], and Gaussian particle swarm optimization (GPSO) [11] or by any hybrid single objective meta-heuristics algorithms such as hybrid particle swarm optimization (PSO) algorithm and firefly algorithm (FA) [12], hybrid ABC algorithm and simulated annealing algorithm (SA) [13], and PSO-GSA algorithm [14].

The third direction is to handle both objectives of CEEDP simultaneously, by using meta-heuristics-based MOO techniques, as competing objective functions instead of transforming the MOP formulation to a single objective problem, as dynamic random neighborhood PSO (DRNPSO) [15], nondominated sorting genetic algorithm (NSGA) [16], niched Pareto genetic algorithm (NPGA) [17], fuzzy clustering-based particle swarm (FCPSO) [18], modified shuffled frog leaping algorithm (MSFLA) [19], real coded genetic algorithm (RCGA) [20], and strength Pareto evolutionary algorithm (SPEA) [21]. The implementation and development of evolutionary-based multiobjective algorithms have significantly grown since they employ a population of individuals in their search. On the other hand, multiple Pareto optimal frontiers can be detected in one single run instead of running the algorithm many times to get multiple Pareto optimal frontiers. These algorithms can be robustly implemented to overcome most of the disadvantages of traditional methods [22].

Due to the importance of CEEDP, researchers have recently proposed new methods for solving CEEDP. In [23], the authors seek to find the best compromise alternative using the quantum genetic algorithm. In [24], Mellal and Williams proposed a hybrid cuckoo optimization algorithm with the binary approach and penalty function to solve CEEDP. In [25], Kim et al. presented a neural network approach to find the optimal solution of CEEDP. In [26], Sundaram proposed a chaotic-based approach to explore the search space to deal with CEEDP. In [27], a novel approach, based on a hybrid algorithm combining a genetic algorithm and a modified Hooke and Jeeves method, was presented to solve the CEEDP with equality constraints. In [28], a novel MOP was proposed to address the problems of economic dispatch and power shedding at the same time. In [29], CEEDP was formulated as a MOP in which the two goals, fuel cost, and emissions of pollutants were optimized at the same time as meeting constraints and addressed by evolutionary multiobjective algorithms. In addition, an empirical study of constraint management has been discussed. In [30], an ameliorated grey wolf optimization algorithm is proposed to solve the CEEDP that coordinates the behavior of grey wolves, random exploration, opposition learning, and local random search. Furthermore, a new paradigm of the CEEDP-based MOP is proposed in [31], where CEEDP has been solved by relatively recent multiobjective algorithms. In [32], Sundaram and Erdogmus proposed a hybrid evolutionary multiobjective optimization system using nondominated sorting genetic algorithm II (NSGA II) and multiobjective PSO to solve the CEEDP. The hybrid approach with a constraint management system is capable of balancing the tasks of exploration and exploitation. In [33], an interesting algorithm, biogeographybased learning particle swarm optimization (BLPSO), was applied to solve the CEEDP with different types of constraints. Simulation results of BLPSO overcome local trap and improve the convergence of the solution. In [34], the authors presented a deterministic optimization based on the augmented Lagrangian to solve CEEDP. In [35], the authors reformulated centralized CEEDP into a decentralized model. In [36], Srivastava and Das presented a new evolutionary-based metaheuristic method, which implemented to solve CEEDP. The performance of the suggested algorithm was evaluated by using twenty-nine benchmark functions and CEEDP. Finally, Zhang et al. [37] presented a decentralized power system for the combined economic emission dispatch problem (CEEDP) model.

One of the key challenges in the solving of constrained MOPs is the handling of constraints. The constraints management strategies are different for the meta-heuristic algorithms [38]. There are four common methods for constraint handling:

(i) Discarding unfeasible options

(ii) The inclusion of a penalty function to reduce the fitness of infeasible solutions

(iii) The development of algorithm mechanism to always generate feasible solutions

(iv) Repairing the unfeasible solutions to be feasible

Handling strategies of constraints i, iii, and iv are specifically applicable to the case of multiobjective. On the other hand, constraint-handling strategy (ii) is not applied explicitly to multiobjective algorithms, due to the fact that the fitness assignment relies on the nondominance rank of the solution, not on the objective function values of the solution [39].

There are several new methods for constraint management that have recently been suggested, such as a modern methodology of constraint management that consistently takes into account proximity, diversity, and feasibility [40], dynamic constraint-handling mechanism [41], adaptive repair method for constraint handling [42], etc. Also, in [43], three typical constraint-handling approaches were implemented into a multiobjective PSO algorithm: (1) dominance of feasible solutions; (2) penalty function; and (3) multiobjective optimization strategy, where the constraints are treated as additional objectives to be optimized.

The Pareto front of MOPs consists of a very large set of Pareto solutions. Selecting a single alternative from this huge set is potentially intractable for any decision-maker (DM) [44]. In order to allow the DM to classify and coordinate solutions, some means of reducing/organizing the set of nondominated solutions are implemented to shrink the size of the Pareto optimal set, which facilitates finding the optimal operating alternative [45]. Several studies have concerned this issue by implementing filtering and cluster analysis to minimize the optimum size of the Pareto to a rational size, enabling the DM to choose the best compromise solution [46]. Algorithms of clustering can be divided into three categories: density-based clustering, 
hierarchical clustering, and centroid-based clustering (partitioning clustering methods) [47]. One of the most common partitioning clustering methods is the K-means, where it is distinguished by its efficiency and robustness in clustering data analysis [48].

There are many meta-heuristics algorithms that have been proposed to solve optimization problems such as genetic algorithms (GA) [49], artificial immune system [50], particle swarm optimization (PSO) [51], ant colony optimization (ACO) [52], artificial bee colony (ABC) [53], bacterial foraging algorithm (BFA) [54], cat swarm optimization (CSO) [55], glowworm swarm optimization algorithm (GSOA) [56], firefly optimization algorithm (FOA) [57], monkey algorithm (MA) [58], krill herd algorithm (KHA) [59], cuckoo search algorithm (CSA) [60], whale optimization algorithm (WOA) [61], sine cosine algorithm (SCA) [62], grasshopper optimization algorithm (GOA) [63], salp swarm algorithm [64], equilibrium optimizer algorithm (EOA) [65], gradient-based optimizer (GBO) [66], slime mould algorithm (SMA) [67], and Harris Hawks optimization (HHO) [68].

As a new algorithm, the equilibrium optimizer (EO) mimics physics-based dynamic source and sink models used to estimate and consider equilibrium states. In this paper, a constrained multiple objective equilibrium optimizer algorithm (EOA) is proposed to solve the combined economic emission dispatch problem (CEEDP) in the power system by modifying the mechanism of the EOA. The proposed methodology is tested by the standard IEEE 30-bus system with six generator units to validate the robustness of the proposed algorithm. In addition, a clustering strategy is used to choose the most compromised set of alternatives based on DM preferences. This paper's major contributions are as follows:

(1) A new approach based on an EOA to solve CEEDP is introduced and tested

(2) Clustering was performed in order to minimize the Pareto border to an appropriate size

(3) A repair algorithm was used to tackle the constraints and unfeasible solution

(4) The robustness and reliability of the proposed algorithm to generate a well-distributed Pareto frontier is tested by the IEEE standard 30-bus system with 6 generators

(5) Numerical analysis results validate the high performance of the proposed algorithm

This paper is organized as follows: MOP formulation is presented in Section 2. The formulation of the combined economic emission dispatch problem is described in Section 3. A constrained multiobjective EOA is presented in Section 4. The simulation analysis is analyzed in Section 5. Finally, the conclusion is presented in Section 6 .

\section{Multiobjective Optimization Problem}

A broad range of applications in architecture, computer science, and many other fields include optimizing several objectives at the same time [69]. In multiobjective optimization problem (MOP), there is no way to decide the best solution where a set of alternatives with different trade-offs among the multiple objectives is retained, which is known as Pareto optimal frontier, instead of getting a single alternative. In the following, the MOP is presented with several general notes and concepts [70].

Definition 1 (formulation of multiobjective optimization problem). Formally, a MOP is stated as follows:

$$
\begin{aligned}
& \text { Min } f(x)=\left[f_{1}(x), f_{2}(x), \ldots, f_{m}(x)\right]^{T}, \\
& \text { s.t. } x \in X .
\end{aligned}
$$

The vector $x \in \mathbb{R}^{n}$ is defined as a vector of $n$ decision variables in the optimization problem formulation. The feasible set $X \subseteq \mathbb{R}^{n}$ is implicitly determined by a set of constraints. The vector function $f: \mathbb{R}^{n} \longrightarrow \mathbb{R}^{m}$ is defined by $m$ scalar objective functions.

Definition 2 (Pareto dominance). Vector $z^{1}$ Pareto-dominates vector $z^{2}$, which is denoted by $z^{1} \succ_{\text {pareto }} z^{2}$, if and only if

$$
\begin{aligned}
& \forall_{i} \in\{1, \ldots, m\}: z_{i}^{1} \leq z_{i}^{2}, \\
& \exists_{i} \in\{1, \ldots, m\}: z_{i}^{1}<z_{i}^{2},
\end{aligned}
$$

i.e., the vector $z^{1}$ Pareto-dominates vector $z^{2}$ if the previous two conditions are satisfied; thus, in order to solve MOP, the set of solutions $x \in X$ should be found whose images $z=$ $f(x)$ are not dominated (nondominated set) by any other vector in the feasible space.

Definition 3 (Pareto optimality concept). A feasible solution $x^{*} \in X$ is a Pareto optimal solution if there does not exist any feasible solution $x \in X$ such that $f(x) \prec_{\text {pareto }} f\left(x^{*}\right)$.

Definition 4 (the set of Pareto optimal solutions). The Pareto optimal set, PO*, is the set, which is defined as

$$
\mathrm{PO}^{*}=\{x \in X \mid \text { ó } \in \mathrm{X}: \mathrm{f}(\mathrm{y}) \prec \text { Pareto } \mathrm{f}(\mathrm{x})\} \text {. }
$$

Definition 5 (Pareto optimal frontier). For a Pareto optimal set, $\mathrm{PO}^{*}$, the Pareto optimal frontier, $\mathrm{PF}^{*}$, is stated as

$$
\mathrm{PF}^{*}=\left\{f(x)=\left(f_{1}(x), \ldots, f_{m}(x)\right) \mid x \in \mathrm{PO}^{*}\right\} .
$$

Definition 6 (ideal objective vector). This vector $z_{i}^{I}$ is a vector whose components are the optimal/minimal values of each objective. The component of $z^{I} \in R^{m}$ can be got by minimizing every objective separately subject to the set of constraints, that is,

$$
\begin{aligned}
z_{i}^{I}= & \operatorname{Min} f_{i}(x), \\
& \text { s.t. } x \in X \text { for } i=1, \ldots, m .
\end{aligned}
$$

By using the ideal vector, the lower limits of the Pareto optimal frontier for each objective function are determined. 
Definition 7 (nadir objective vector) [71]. Nadir vector is the upper bound of the optimal Pareto frontier. The nadir objective vector components $z^{\text {nad }}$ can be estimated from the payoff table as depicted in Figure 1.

\section{The Formulation of Combined Economic Emission Dispatch Problem}

The CEEDP involves the optimization of two multiple conflicting objectives, generation operation cost, and pollutant emission, which must be addressed at the same time. The problem formulation is as follows.

3.1. Objective Functions. The formulation of the objective functions of the problem, which are objective for the economy and environmental objective, is seen in this subsection.

3.1.1. Generation Operation Cost (Economy Objective). The traditional CEEDP problem is to decide the optimum active power produced by the power system with minimizing the total cost of the generation operation and satisfying the overall load requirement. The economy objective can be formulated mathematically as [72]

$$
\begin{aligned}
f_{1}\left(P_{G i}\right)=C_{t}=\sum_{i=1}^{n} C_{i}\left(P_{G i}\right)= & \sum_{i=1}^{n}\left(a_{i}+b_{i} P_{G i}+c_{i} P_{G i}^{2}\right) \\
& +e_{i} \sin \left(f_{i}\left(P_{G i}-P_{G i, \min }\right)\right) \$ / h,
\end{aligned}
$$

where $C_{t}$ is the overall cost of fuel that is used for generation operations $(\$ / h)$, the parameters $a_{i}, b_{i}, c_{i}, e_{i}, f_{i}$ are the coefficients of the cost for each generator $i, P_{G i}$ is the generated active power (p.u) by each generator $i, P_{G i \text {, min }}$ is the minimal generation limit of unit $i$, and $n$ is the generator numbers. The valve point loading effect is overlooked in this study.

3.1.2. Pollutant Emission (Environmental Objective). The other environmental objective is the pollutant emission, which can be defined as the amount of all types of pollutant emission, such as nitrogen oxides $\left(\mathrm{NO}_{x}\right)$ and sulfur dioxide 2 $\left(\mathrm{SO}_{2}\right)$. In this study, only "nitrogen oxide" pollutant emissions are taken into account without a lack of generality. As a quadratic and exponential function, the amount of emission pollutants is indicated as

$$
f_{2}\left(P_{G i}\right)=E_{\mathrm{NO}_{x}}=\sum_{i=1}^{n}\left[\alpha_{i}+\beta_{i} P_{G i}+\gamma_{i} P_{G i}^{2}+\xi_{i} e^{\left(\lambda_{i} P_{G i}\right)}\right]\left(\frac{\text { ton }}{h}\right),
$$

where the parameters $\alpha_{i}, \beta_{i}, \gamma_{i}, \xi_{i}, \lambda_{i}$ are the coefficients of each generator's $\mathrm{NO}_{x}$ emission.

3.2. Constraints. The formulation of CEEDP is restricted by a set of constraints as follows.

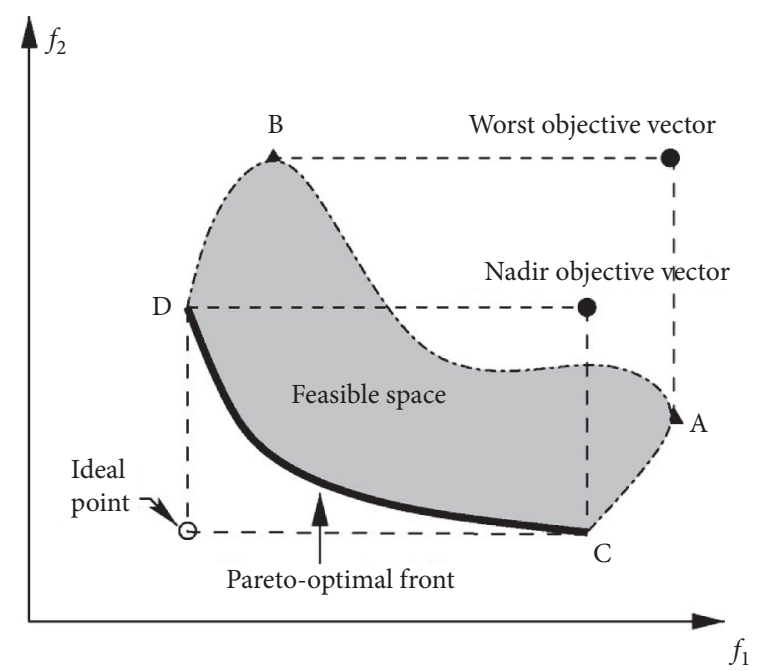

FIGURE 1: Ideal, nadir, and worst objective vectors.

3.2.1. The Set of Active Power Constraints. The total active power constraints delivered by the generator must provide the total load demand $P_{D}$ and the transmission line losses $P_{\text {loss: }}$ :

$$
\sum_{i=1}^{n} P_{G i}-P_{\text {loss }}=P_{D}
$$

where $P_{D}$ is the total load demand (p.u.) and $P_{\text {loss }}$ is the total transmission line losses (p.u.). [73]:

The losses of the transmission line are given as follows

$$
\begin{aligned}
P_{\text {loss }} & =\sum_{i=1}^{n} \sum_{i=1}^{n}\left[A_{i j}\left(P_{i} P_{j}+Q_{i} Q_{j}\right)+B_{i j}\left(Q_{i} P_{j}-P_{i} Q_{j}\right)\right], \\
P_{i} & =P_{G i}-P_{D i} \\
Q_{i} & =Q_{G i}-Q_{D i}, \\
A_{i j} & =\frac{R_{i j}}{V_{i} V_{j}} \cos \left(\delta_{i}-\delta_{j}\right), \\
B_{i j} & =\frac{R_{i j}}{V_{i} V_{j}} \sin \left(\delta_{i}-\delta_{j}\right),
\end{aligned}
$$

where the system parameters are defined as follows:

(i) $n$ is the number of buses,

(ii) $R_{i j}$ is the series resistance linking buses $i$ and $j$,

(iii) $V_{i}$ is the voltage magnitude at bus $i$,

(iv) $\delta_{i}$ is the voltage angle at bus $i$,

(v) $P_{i}$ is the real active power injection at bus $i$, and

(vi) $Q_{i}$ is the reactive power injection bus $i$. 
3.2.2. Upper Bound and Lower Bound of Active Power Generation. The active power $P_{G i}$ delivered by each generator in the power system is constrained by its upper bound and lower bound, i.e.,

$$
\begin{aligned}
P_{G i \min } & \leq P_{G i} \leq P_{G i \max }, \\
Q_{G i \min } & \leq Q_{G i} \leq Q_{G i \max }, \\
V_{i \min } & \leq V_{i} \leq V_{i \max }, \quad i=1, \ldots, n,
\end{aligned}
$$

where

(i) $P_{G i \text { min }}$ is the lower bound active power generated, and

(ii) $P_{G i \max }$ is the upper bound active power generated.

3.2.3. The System Security Constraints. The CEEDP formulation shall take into account the limited proportion of system lines in violation or in near violation of security limits; these lines are known as the critical lines. The system critical lines that connect in the optimal solution are only taken into account. The experiences of the DM help to detect the critical lines. The protection limit can be maintained by minimizing the following equation:

$$
S=f\left(P_{G i}\right)=\sum_{j=1}^{k} \frac{\left|T_{j}\left(P_{G}\right)\right|}{T_{j}^{\max }},
$$

where

(i) $T_{j}\left(P_{G}\right)$ is the real active power flow,

(ii) $T_{j}^{\max }$ is the maximum limit of the active power flow through the $j^{\text {th }}$ line, and

(iii) $k$ is the number of critical lines.

The line active power flow through the $\mathrm{j}^{\text {th }}$ line is defined in terms of the control variables $P_{G s}$, by implementing the generalized generation distribution factors (GGDFs) [74], and is defined as follows:

$$
T_{j}\left(P_{G}\right)=\sum_{i=1}^{m}\left(D_{i j} P_{G i}\right),
$$

where the parameter $D_{i j}$ is the GGDF for line $j$, due to generator $i$. For protection, the loading of the transmission line $S_{l}$ is limited by its maximum, which is given as $S_{l} \leq S_{l \max }, l=1, \ldots, m_{l}$, where $m_{l}$ is the number of transmission line in the power system.

\section{Constrained Multiobjective Equilibrium Optimizer Algorithm}

In this section, we introduce a novel evolutionary-based algorithm depending on the EOA [65] which simulates the equilibrium and dynamic $m$ states related to the mass balance models.

4.1. Brief Introduction to Equilibrium Optimizer Algorithm. The equilibrium optimizer algorithm (EOA) is an optimizer that was firstly presented by Farmarzi [65] which first appeared in 2020. It simulates the balance and dynamic states associated to models for mass balance, in which each position (concentration of particles) is randomly updated in order to reach equilibrium state (fitness). The equilibrium optimizer is easy to use. Also, it has an adaptive dynamic control parameter. EOA is started with the initial particle positions (initial population $C_{i}, i=1,2, \ldots$, no. of particles) and problem's dimensions (dim) as in the following equation:

$$
C_{\text {initial }}=\operatorname{rand}(\text { search particles_no, } \operatorname{dim}) \times(u b-l b)+l b,
$$

where $C_{\text {initial }}$ locates the initial positions of the particles; the bounds lband ubare the specified lower and upper bounds respectively of the decision optimization variables.

4.1.1. Equilibrium Pool and Candidates (Ceq). The equilibrium/balance state is the EOA's final state of convergence. At the beginning of the algorithm, equilibrium candidates are allocated to support a particle search pattern. During the optimization process, the four best so-far particles are determined in addition to another particle whose concentration is the arithmetic mean of the four particles. EOA has an exploration scheme using four candidates and an exploitation scheme using the average mean. For constructing a vector called the balance pool, these five particles are called candidates of equilibrium:

$$
C_{\text {eq,pool }}=\left(C_{\text {eq, } 1}, C_{\text {eq }, 2}, C_{\text {eq }, 3}, C_{\text {eq }, 4}, C_{\text {eq,av }}\right) .
$$

The position of each particle in each iteration, by the same probability, is updated using the random selection among the chosen candidates. Then, the particle positions are regularly updated. The updating procedure of the EOA is as in the following equation:

$$
\begin{aligned}
C_{\text {new }} & =C_{\text {eq }}+\frac{G}{\lambda}(1-F)+\left(C_{\text {old }}-C_{\text {eq }}\right) \times F, \\
F & =a_{1} \operatorname{sign}\left(r_{1}-0.5\right)\left(e^{-\lambda t}-1\right), \\
G & = \begin{cases}0.5 r_{1}, & \text { if } r_{2} \geq G P, \\
0, & \text { if } r_{2}<G P,\end{cases} \\
t & =\left(1-\frac{T}{T_{\max }}\right)^{a_{2}\left(T / T_{\max }\right)},
\end{aligned}
$$

where $C_{\text {old }}$ is the current position vector and $C_{\text {new }}$ is the new position vectors of the particle. From the equilibrium pool, we pick one concentration vector randomly which nominated by $C_{\mathrm{eq}} \cdot \lambda$ is a random vector between 0 and $1 ; a_{1}$ and $a_{2}$ are constants $\left(a_{1}=2\right.$ and $\left.a_{1}=1\right), r, r_{1}, r_{2}$ are random numbers between 0 and $1, T$ is the current iteration counter, and $T_{\max }$ is the number of iterations maximum. In each iteration, for each particle's position, the considered objective function is measured to estimate its status. The 
equilibrium pool is also updated to include the four best particles to date in every iteration.

4.2. Dominance Criteria. To improve the EOA to deal with MOPs, we incorporate the dominance criteria in the proposed algorithm, which are described as follows:

For any problem having multiple objective functions (say $f_{j}, j=1, \ldots, k, k>1$ ), any two particles $x^{1}$ and $x^{2}$ can have one of two cases, "one dominates the other" or "none dominates the other."

The particle $x^{1}$ dominates the particle $x^{2}$, if the following two conditions are satisfied [75]. Note that the operator $<$ denotes worse and the operator $>$ denotes better.

(1) The particle $x^{1}$ is no worse than $x^{2}$ in all objectives, or $f_{j}\left(x^{1}\right) \nprec f_{j}\left(x^{2}\right), \quad \forall j=1, \ldots, p$.

(2) The particle $x^{1}$ is strictly better than $x^{2}$ in at least one objective, or $f_{j}\left(x^{1}\right)>f_{j}\left(x^{2}\right)$ for at least one $j \in\{1,2, \ldots, k\}$.

If any of the above two conditions is infringed, the particle $x^{1}$ dose not dominate the particle $x^{2}$. This algorithm is repeatedly for all populations, all particles that are not dominated by any other individuals are from "the nondominated set".

4.3. Archive/Selection Strategy. The vector function $F$ is represented by $m$-dimensional, where each coordinate is one of the objectives (Figure 2). In each iteration counter $t$, the aim of the archive/selection technique is to create a new set of solutions. This technique uses the old archive set $A^{(t-1)}$ to update the archive content $A^{(t)}$. Overall, the purpose of this archive is to collect useful data about optimization problems during the run and update the content of the stored data.

4.4. Identifying the K-Centroids of Pareto Front. The solution of MOP by using the concept of dominance produces a large size of Pareto optimal solutions, not a single elite optimum solution [76]. The selection of a solution to achieve various objectives is the most critical task in practical engineering applications. Also, decreasing the size of the Pareto set, that is available to the DM to select, saves effort and time. Cluster analysis has been implemented to shrink the volume of the Pareto frontier to a reasonable volume using a predetermined size. $K$-means is an important partitioning clustering which is used to classify $n$ observations into $K$ clusters. Consider the Pareto front which is $N$ points with $m$ dimensions, where $m$ is the number of objectives. The following steps are used to identify the $K$-centroids of the Pareto front:

(i) Step 1: the initial $K$ cluster centers $C e_{1}, C e_{2}, \ldots, C e_{K}$ are randomly selected from the Pareto frontier $P f^{*}=\left\{P f_{1}^{*}, P f_{2}^{*}, \ldots, P f_{N}^{*}\right\}$.

(ii) Step 2: each agent form the Pareto set $P f_{i}^{*}, \quad i=$ $1,2, \ldots, N$ is allocated to cluster $C_{j}, \quad j \in\{1,2, \ldots, k\}$ if and only if

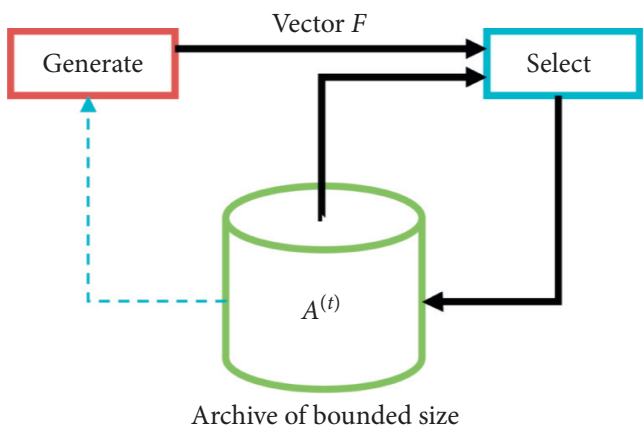

FIGURE 2: Block structure of archive-based selection algorithm.

$$
\left\|P f_{i}^{*}-C e_{j}\right\|<\left\|P f_{i}^{*}-C e_{p}\right\|, \quad p=1,2, \ldots, K \& j \neq p .
$$

(iii) Step 3: centers of new cluster $C e_{1}, C e_{2}, \ldots, C e_{K}$ are determined as follows:

$$
C e_{i}^{*}=\frac{1}{n e_{i}} \sum_{P f_{j}^{*} \in C_{i}} P f_{j}^{*}, \quad \forall i=1,2, \ldots, K,
$$

where $n e_{i}$ is the elements number of the cluster $C_{j}$.

(iv) Step 4: if $C e_{i}^{*}=C e_{i}, \quad \forall i=1,2, \ldots, K$, then the algorithm stops; otherwise, go to Step 2.

4.5. Basic Algorithm. The structure of the proposed algorithm is shown in Figure 3. In order to guarantee the obtaining for the true Pareto optimal set, Figure 3 shows how the Pareto set is updated and how it is applied in the procedure of proposed algorithm. In addition, an archiving-based selection algorithm was used to ensure retaining the Pareto optimal frontier and monitoring of the whole domain of the nondominated set. On the other hand, the violation in the constraint will be handled using a repair method [77], where it separates and repairs any infeasible particle in population at each generation. Firstly, an initial reference point $R$ (feasible reference point) is defined for the repair phase. Infeasible particles in the population are then repaired according to the repair process until they are feasible. The repair process produces a new viable particle $(z)$ instead of an unfeasible one $(q)$ on a segment identified by the two points $(R$ and $q)$. This segment can be expanded equally by a specified parameter $\mu \in[0,1]$ on both sides. Therefore, the new feasible particle $z$ is generated as

$$
z=\left\{\begin{array}{l}
\gamma \cdot q+(1-\gamma) \cdot R \\
\gamma \cdot R+(1-\gamma) \cdot q
\end{array}\right.
$$

where $\gamma=(1+2 \mu) \delta-\mu$ and $\delta \in[0,1]$ is a random number. Figure 4 shows a schematic view of the constraint-handling method for the generated particles, finally, defining the $K$ centroids of Pareto front using the $K$-means clustering algorithm. 


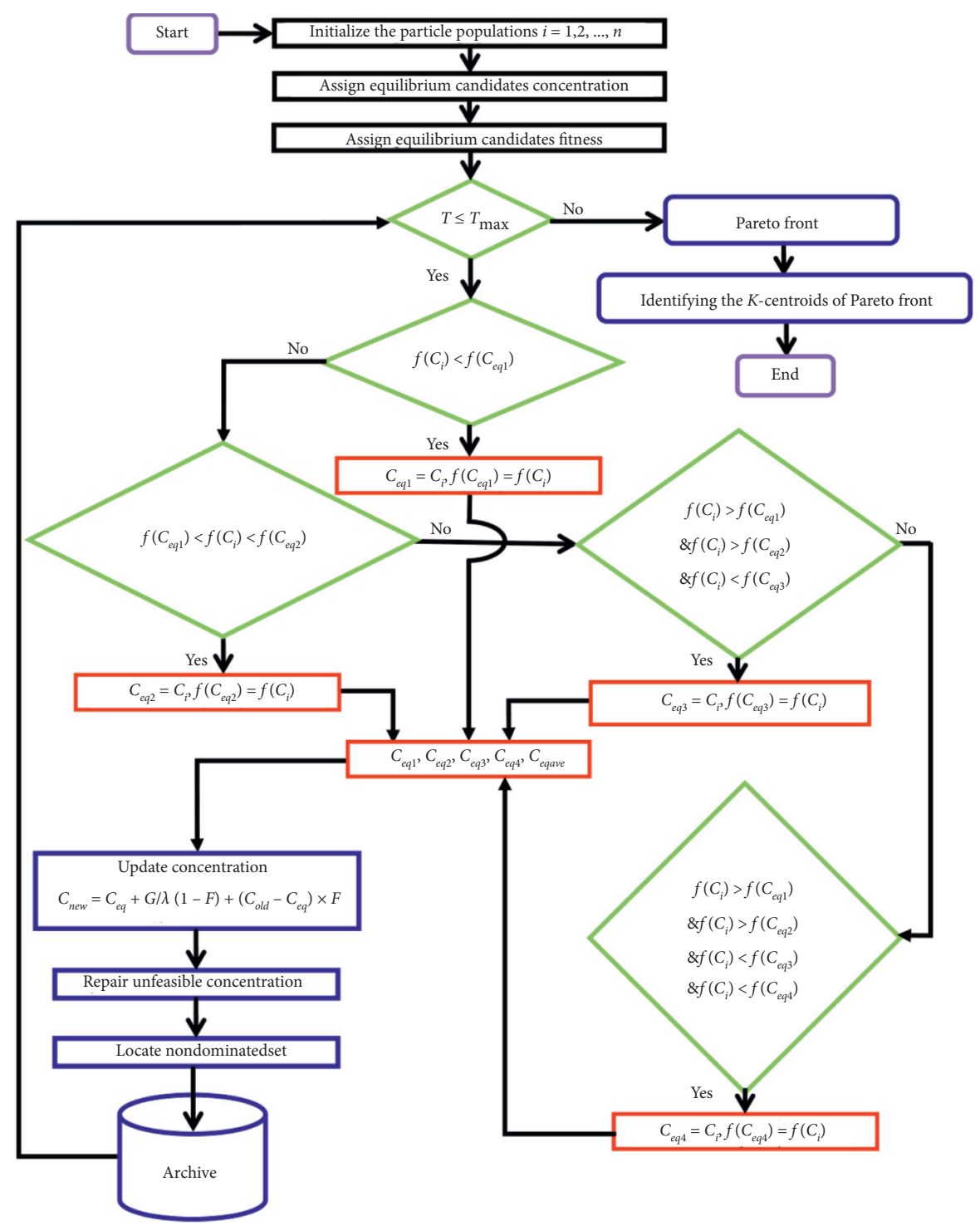

FIgURE 3: The structure of the proposed algorithm.

\section{Simulation Analysis}

The standard IEEE system having a 30-bus system and six generator units is elected to confirm the robustness and the proposed algorithm efficiency. The data of the test system consists of data for running the generators located at buses 25-30 and 41 lines. Figure 5 illustrates the diagram of this system. Fuel cost with the generator's limits and pollutant emission $\mathrm{NO}_{x}$ parameters for this system are shown in Table 1, where the down ramp rate is considered as $10 \%$. The transmission B-loss coefficient matrix is determined by using a load flow software as seen in Table 2, as used in [78]. The overall demand for power is 2.834 p.u. in the system at the base of 100 MVA. All information for this system is given in the MATLAB power system simulation package [79].

The proposed algorithm is coded in MATLAB (R2016b) and implemented on the computer with Intel Core i5, $1.80 \mathrm{GHz}$ and $4 \mathrm{~GB}$ RAM. Like any meta-heuristic

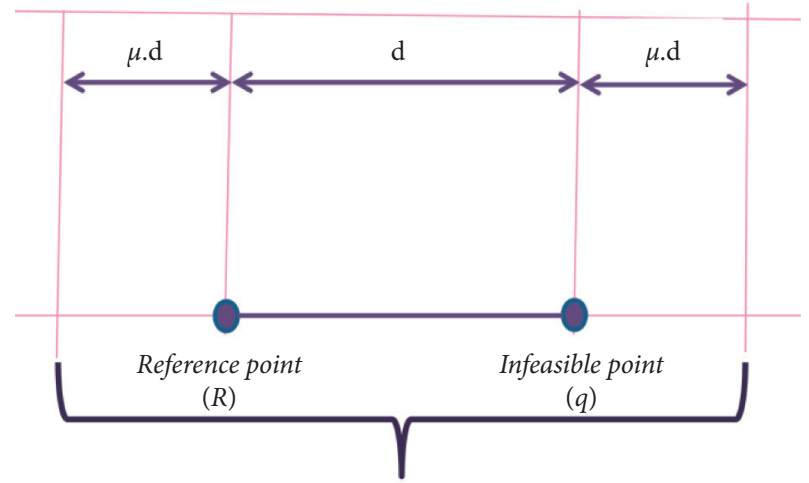

Figure 4: Constraint-handling method.

algorithm, the proposed algorithm requires a set of parameters that influence its performance. The controlled parameters of the proposed algorithm are shown in Table 3. 


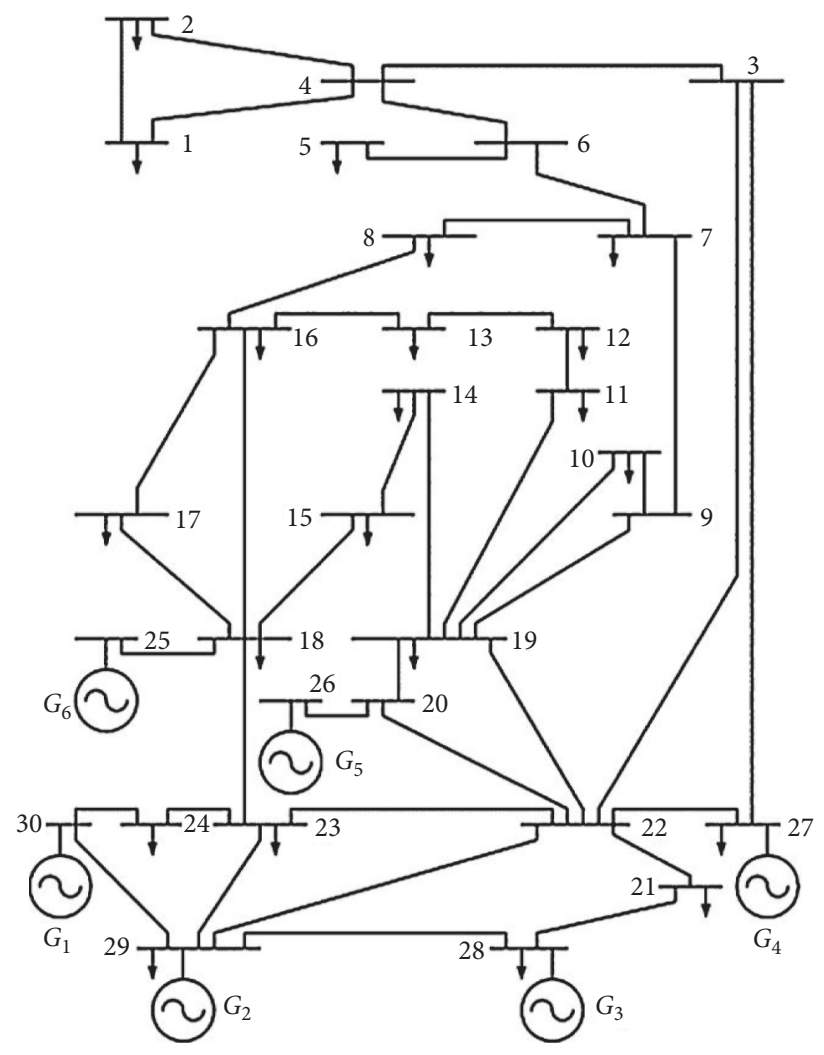

FIgURE 5: IEEE system line diagram of 30-bus 6 generators.

TABLe 1: Generator limits, fuel cost coefficients, and emission coefficients of the IEEE 30-bus system.

\begin{tabular}{lcccccccrrr}
\hline \multirow{2}{*}{ Generator (i) } & \multicolumn{2}{c}{ Generation limits } & \multicolumn{3}{c}{ Fuel cost coefficients } & \multicolumn{3}{c}{ Emission coefficients } \\
& $P_{\text {Gi min }}$ & $P_{\text {Gi max }}$ & $a_{i}$ & $b_{i}$ & $c_{i}$ & $\alpha_{i}$ & $\beta_{i}$ & $\gamma_{i}$ & $\xi_{i} \times 10^{-6}$ & $\lambda_{i}$ \\
\hline 1 & 0.05 & 0.50 & 10 & 200 & 100 & 4.091 & -5.554 & 6.490 & 200 \\
2 & 0.05 & 0.60 & 10 & 150 & 120 & 2.543 & -6.047 & 5.638 & 500 & 3.857 \\
3 & 0.05 & 1.00 & 20 & 180 & 40 & 4.258 & -5.094 & 4.586 & 1.0 & 8.000 \\
4 & 0.05 & 1.20 & 10 & 100 & 60 & 5.326 & -3.550 & 3.380 & 2000 & 2.000 \\
5 & 0.05 & 1.00 & 20 & 180 & 40 & 4.258 & -5.094 & 4.586 & 1.0 & 8.000 \\
6 & 0.05 & 0.60 & 10 & 150 & 100 & 6.131 & -5.555 & 5.151 & 10 & 6.667 \\
\hline
\end{tabular}

TABle 2: Coefficient matrix of transmission B-loss for the test system: IEEE 30 bus.

\begin{tabular}{lcccccc}
\hline & 0.02180 & 0.01070 & -0.00036 & -0.00110 & 0.00055 & 0.00330 \\
& 0.01070 & 0.01704 & -0.00010 & -0.00179 & 0.0026 & -0.01180 \\
$B_{i j}=$ & -0.00040 & -0.00020 & 0.02459 & -0.01328 & 0.00790 \\
& -0.0011 & -0.00179 & -0.01328 & 0.0265 & 0.0045 \\
& 0.00055 & 0.00026 & -0.0118 & 0.0098 & 0.0216 & -0.0001 \\
& 0.0033 & 0.0028 & -0.00792 & 0.0045 & -0.00012 & 0.02978 \\
\hline$B_{0 i}=$ & $1.0731 e-05$ & 0.0017704 & -0.0040645 & 0.0038453 & 0.0055503 \\
\hline$B_{00}=$ & & \multicolumn{3}{c}{0.0014} \\
\hline
\end{tabular}

TABle 3: The proposed algorithm parameters.

\begin{tabular}{lc}
\hline Parameter & Value \\
\hline No. of particles & 50 \\
The iterations maximum number $\left(T_{\max }\right)$ & 100 \\
Probability of generation $(G P)$ & 0.5 \\
$a_{1}$ & 2 \\
$a_{2}$ & 1 \\
Number of clusters $(K)$ & $5-100$ \\
\hline
\end{tabular}


To examine the proposed algorithm, it is implemented to solve CEEDP with the following 3 cases of IEEE system:

(i) Case 1: single objective function, where the fuel cost was minimized as constrained nonlinear programming

(ii) Case 2: single objective function, where the generation emission was minimized as constrained nonlinear programming

(iii) Case 3: minimizing the two objective functions (fuel cost and generation emission) as a MOP using domination criteria

For the first two cases (case 1 and case 2), the obtained results by applying the proposed algorithm were compared to other optimization literature algorithms such as DRNPSO [15], NSGA [16], NPGA [17], FCPSO [18], MSFLA [19], RCGA [20], and SPEA [21] as illustrated in Table 4. From Table 4, it was concluded that the proposed method provided a better minimum fuel cost for case 1 and found minimum generation emissions for case 2 than the other algorithms. For case 3, Figure 6 represents the Pareto optimal set for the CEEDP obtained by the proposed algorithm. From the figure, it can be concluded that the proposed algorithm is capable of getting the Pareto frontier for the CEEDP.

Figure 7 represents the convergence analysis of the proposed method to obtain the optimal solution. Figure 7 (a) represents the cost function convergence versus the generation; it declares that the cost function has converged to its optimal value in the first 50 generations. On the other hand, Figure 7(b) represents the emission function convergence versus the generation; it declares that the emission function has converged to its optimal value in the first 150 generations. Figure 7(c) represents the locus of the ideal points which is starting at point $(631.7933,0.2161)$ and ending at point (599.0424, 0.1928). Finally, Figure 7(d) represents the locus of the nadir points which starts from point $(600.0432,0.1932)$ and ends at point $(0.2204,639.1063)$.

Implementation of dominance criteria provides not a single optimum solution, but a huge set of solutions as in Figure 1. For practical engineering applications, the selection of the best solution or limited set that will satisfy different objectives to some extent is the most important task. Also, minimizing the size of the Pareto set is available to the DM to select and save effort and time. Cluster analysis was implemented to shrink the size of the Pareto optimal set to a small set with a predetermined size $K$ as in Table 5 . Figures 8-11 represent some reduced Pareto sets depending on the chosen $K$ parameter which may save effort to choose the best compromise alternative from the Pareto optimal set.

5.1. Discussion. It is clear from Table 4 that the suggested algorithm has done better than all state-of-the-art approaches in case 1 and case 2. While Figure 6 indicates that the proposed algorithm was able to solve CEEDP as a multiobjective optimization problem and gave a uniform distribution of the Pareto optimal curve and obtained very good solutions at both ends of the curve. Furthermore, Figure 7 indicates the accelerated convergence of the
TABLE 4: Optimal solutions by different methods: case 1 and case 2 .

\begin{tabular}{lcccc}
\hline & \multicolumn{4}{c}{ Cases } \\
Algorithm & $\begin{array}{c}\text { Case 1 } \\
\text { (minimizing fuel } \\
\text { costs) }\end{array}$ & $\begin{array}{c}\text { Case 2 } \\
\text { (minimizing } \\
\text { generation } \\
\text { emissions) }\end{array}$ \\
\hline DRN-PSO [15] & 591.1517 & 0.2150 & 643.8616 & 0.1949 \\
NSGA [16] & 600.3100 & 0.2238 & 633.8300 & 0.1946 \\
NPGA [17] & 600.2200 & 0.2206 & 636.0400 & 0.1943 \\
FCPSO [18] & 600.1300 & 0.2223 & 638.3577 & 0.1942 \\
MSFLA [19] & 600.1114 & 0.2225 & 638.2425 & 0.1942 \\
RCGA [20] & 611.6935 & 0.2285 & 648.5301 & 0.1932 \\
SPEA [21] & 600.3400 & 0.2241 & 640.4200 & 0.1942 \\
Proposed approach & $\mathbf{5 9 9 . 0 4 2 4}$ & 0.2204 & 639.1063 & $\mathbf{0 . 1 9 2 8}$ \\
\hline
\end{tabular}

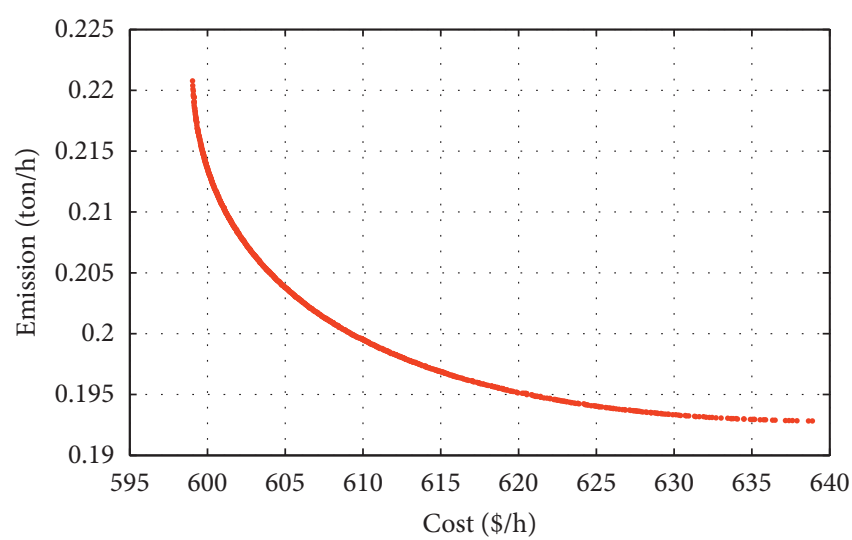

FIgURE 6: CEEDP Pareto optimal set obtained by the proposed algorithm.

proposed algorithm, where the cost function has converged to its optimum value over the first 50 generations and the emission function has converged to its optimum value over the first 150 generations. In addition, Figures 8-11 indicate the potential of the proposed algorithm to help DM find a compromise solution on the whole Pareto front by splitting it into a variety of regions using the K-means clustering algorithm.

A comparative study has been investigated in this subsection to examine the proposed method concerning the assumptions of convexity, the smoothness of the Pareto front, the number of the set of nondominated frontier, the handling constraint, and the closeness to the true Pareto optimal set.

Firstly, unfortunately, the CEEDP is a nonlinear optimization problem and having multimodal functions. Also, it needs many mathematical assumptions such as differential functions, analytics, and convexity. Therefore, traditional optimization techniques implement derivatives and gradients of a function, in general, not valid to detect the optimal solution. On the other hand, the proposed method does not need any assumption regarding derivatives, gradients, and convexity.

Other evolutionary-based algorithms lack the shortage in numbers of nondominated solutions in the Pareto frontier 


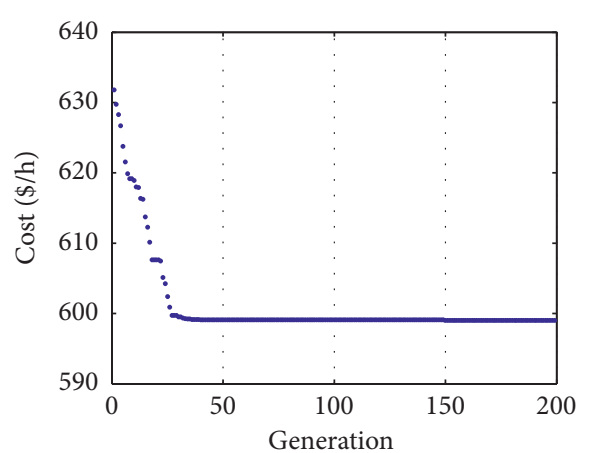

(a)

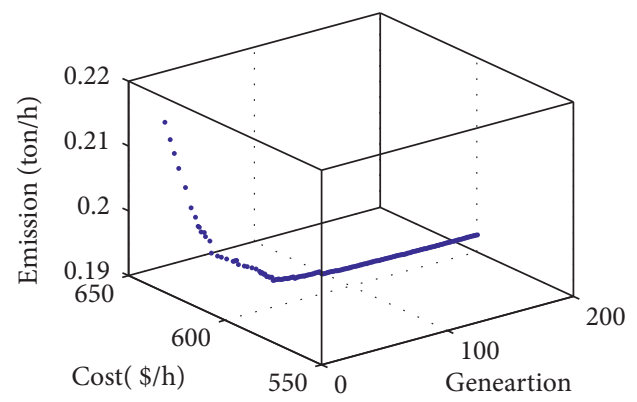

(c)

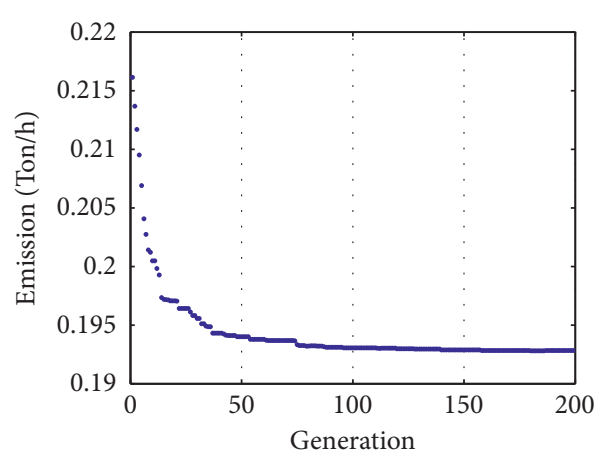

(b)

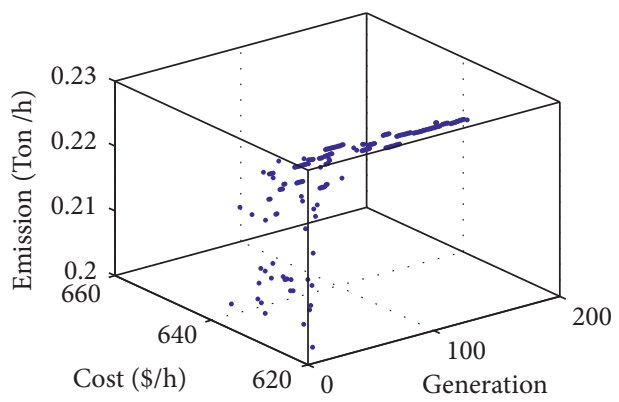

(d)

Figure 7: Pareto optimal convergence. (a) Cost ( $\$ / \mathrm{h})$ versus generation. (b) Emission (Ton/h) versus generation. (c) Ideal points versus generation. (d) Nadir points versus generation.

TABLE 5: Implementation of $K$-means method to reduce the shrink Pareto optimal frontier.

\begin{tabular}{lcr}
\hline$K$ & Size of reduced Pareto set & Ideal point \\
\hline 100 & 100 & $(0.1933,601)$ \\
50 & 50 & $(0.1930,599.8)$ \\
10 & 10 & $(0.1929,599.1)$ \\
5 & 5 & $(0.1929,599.1)$ \\
\hline
\end{tabular}

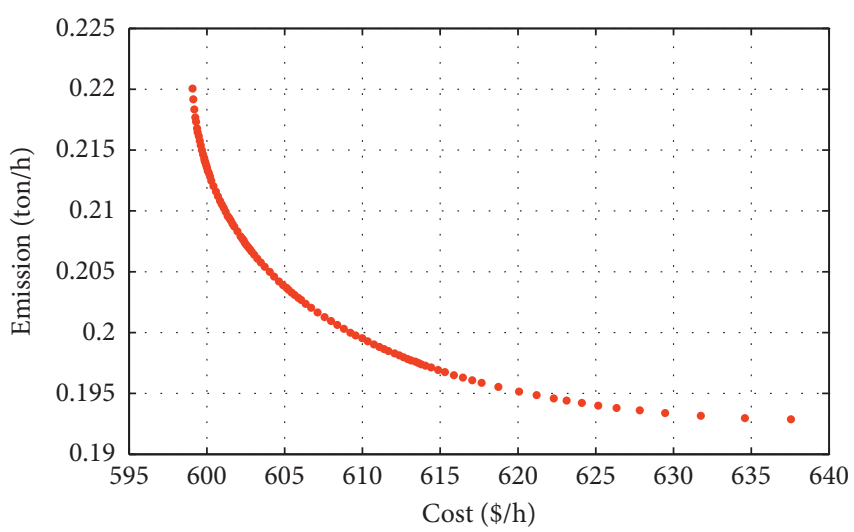

FIGURE 8: Compromising 100 alternatives from the Pareto set.

(does not cover the whole Pareto frontier). On the other side, the proposed method has been effectively exploring the whole search space using dominance criteria and to handle unfeasibility by implementing the repair algorithm which enables the algorithm to deal with constrained optimization.
Also, the proposed algorithm is capable of efficiently discovering a large set of the Pareto front, spanning the whole Pareto optimal frontier.

Secondly, the proposed method keeps track of all nondominated solutions detected during the process of optimization (i.e., the algorithm has no restriction on the number of Pareto optimal solutions), by using an archivingbased selection technique that ensures the convergence towards the Pareto optimal set. On the other hand, the proposed algorithm is a meta-heuristic-based multiple-objective optimization method where it uses a population of particles in its search. So, multiple solutions (Pareto optimal) can be found in one single run. On the contrary to traditional techniques, which require a lot of runs to get the Pareto optimal solutions.

Furthermore, it is observed from the simulation analysis that the proposed method is absolutely better than other algorithms, where its behavior is completely good and it is capable of retaining the Pareto frontier for the CEEDP problem. Finally, unlike other methods, the proposed method does not leave the DM confused in front of choosing a suitable solution from among all the 


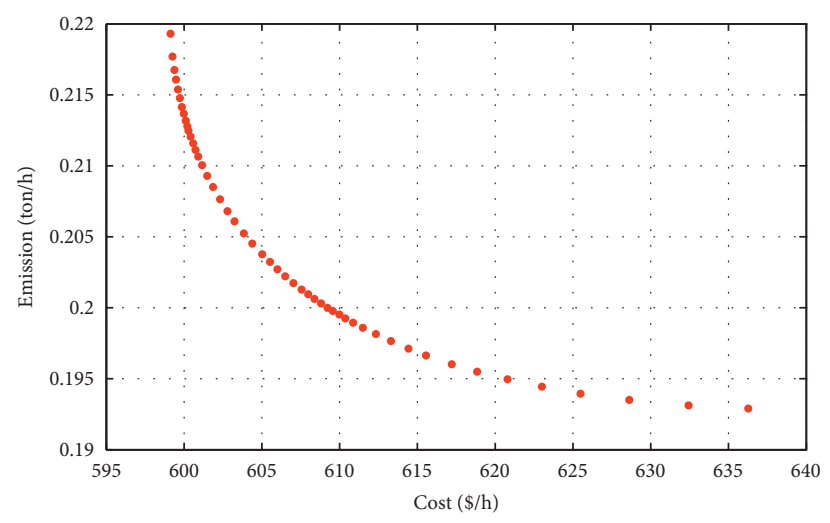

Figure 9: Compromising 50 alternatives from the Pareto set.

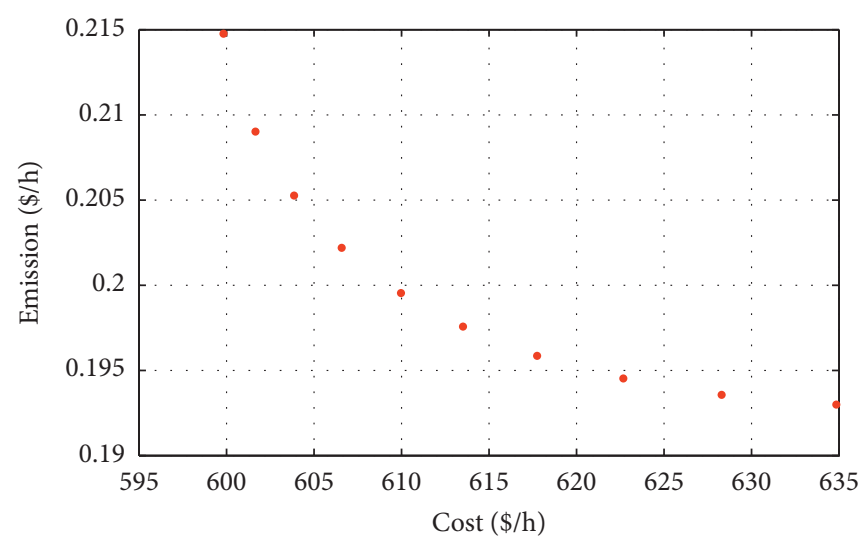

FIgUre 10: Compromising 10 alternatives from the Pareto set.

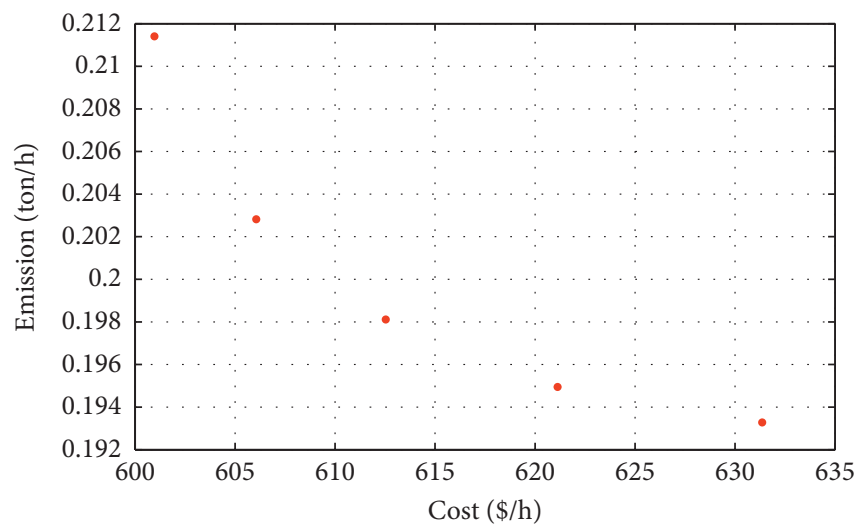

FIgURE 11: Compromising 5 alternatives from the Pareto set.

nondominated sets obtained but divides the Pareto front into a set of clusters with a center for each cluster by one of the most famous methods of cluster analysis, namely, $K$ means.

\section{Conclusion}

This paper investigates a new optimization system called constrained multiobjective equilibrium optimizer for dealing with the constrained combined economic emission dispatch problem (CEEDP) with two objectives that reflect minimizing the generation cost and minimizing the environmental pollution emission. The proposed algorithm incorporates the dominance criteria to handle the multiobjective functions that enable the DM to detect all the Pareto frontiers. Cluster analysis was implemented to decrease the size of the Pareto frontier to a reasonable size that reflects all the characteristics of the original Pareto frontier. On the other hand, the repair method was applied to handle the constraints and feasibility of particles. Solving the 
standard 30-bus IEEE system demonstrates the superiority of the proposed algorithm to generate a well-distributed Pareto frontier.

The following points are the major contribution points of this research:

(a) The proposed algorithm was applied efficiently to deal with constrained multiobjective CEEDP effectively, without limiting the treatment of more than two objectives

(b) The obtained Pareto optimal frontier is well distributed

(c) By means of cluster analysis, the proposed algorithm according to DM needs help to track the resolution of the Pareto frontier

(d) Incorporating the repair method enables the algorithm to retain the particles feasibility follows:

This paper can be developed as a future research study as

(i)Constrained multiobjective equilibrium optimizer algorithm used in this analysis also needs further testing and development and can also be paired with other approaches to further improve its efficiency

(ii) Economic dispatch issues in this analysis may be expanded to cover losses in repairing engine and operating costs

\section{Data Availability}

All data used to support the findings of this study are included within the article.

\section{Conflicts of Interest}

The authors declare that this article content has no conflicts of interest.

\section{Acknowledgments}

The authors extend their appreciation for the great support of Deanship of Scientific Research, Taif University, for funding Taif University Researcher Supporting Project Number Tursp-2020/48, Taif University, Taif, Saudi Arabia.

\section{References}

[1] S. F. Brodesky and R. W. Hahn, "Assessing the influence of power pools on emission constrained economic dispatch," IEEE Transactions on Power, vol. 1, no. 1, pp. 57-62, 1986.

[2] M. S. Osman, M. A. Abo-Sinna, and A. A. Mousa, "An $\varepsilon$-dominance-based multiobjective genetic algorithm for economic emission load dispatch optimization problem," Electric Power Systems Research, vol. 79, no. 11, pp. 1561-1567, 2009.

[3] M. A. Hussein, A. A. EL-Sawy, E. L.-S. M. Zaki, and A. A. Mousa, "Genetic algorithm and rough sets based hybrid approach for economic environmental dispatch of power systems," British Journal of Mathematics \& Computer Science, vol. 4, no. 20, pp. 2978-2999, 2014.

[4] A. Farag, S. Al-Baiyat, and T. C. Cheng, "Economic load dispatch multiobjective optimization procedures using linear programming techniques," IEEE Transactions on Power Systems, vol. 10, no. 2, pp. 731-738, 1995.

[5] C. S. Chang, K. P. Wong, and B. Fan, "Security-constrained multiobjective generation dispatch using bicriterion global optimisation," IEEE Proceedings-Generation, Transmission and Distribution, vol. 142, no. 4, pp. 406-414, 1995.

[6] J. X. Xu, C. S. Chang, and X. W. Wang, "Constrained multiobjective global optimisation of longitudinal interconnected power system by genetic algorithm," IEE Proceedings Generation, Transmission and Distribution, vol. 143, no. 5, pp. 435-446, 1996.

[7] Y. T. Hsiao, H. D. Chiang, C. C. Liu, and Y. L. Chen, "A computer package for optimal multi-objective VAR planning in large scale power systems," IEEE Transactions on Power Systems, vol. 9, no. 2, pp. 668-676, 1994.

[8] B. S. Kermanshahi, Y. Wu, K. Yasuda, and R. Yokoyama, "Environmental marginal cost evaluation by non-inferiority surface (power systems)," IEEE Transactions on Power Systems, vol. 5, no. 4, pp. 1151-1159, 1990.

[9] S. Mouassa and T. Bouktir, "Artificial bee colony algorithm for solving economic dispatch problems with non-convex cost functions," International Journal of Power and Energy Conversion, vol. 8, no. 2, pp. 146-165, 2017.

[10] R. K. Swain, N. C. Sahu, and P. K. Hota, "Gravitational search algorithm for optimal economic dispatch," Procedia Technology, vol. 6, pp. 411-419, 2012.

[11] S. Komsiyah, "Computational methods of Gaussian particle swarm optimization (GPSO) and lagrange multiplier on economic dispatch issues (case study on electrical system of Java-Bali IV area)," The European Physical Journal, EDP Sciences, vol. 68, , 2014.

[12] S. Arunachalam, T. AgnesBhomila, and M. Ramesh Babu, "Hybrid particle swarm optimization algorithm and firefly algorithm based combined economic and emission dispatch including valve point effect," in Proceedings of the 5th International Conference on Swarm, Evolutionary, and Memetic Computing, December 2014.

[13] S. Arunachalam, R. Saranya, and N. Sangeetha, "Hybrid artificial bee colony algorithm and simulated annealing algorithm for combined economic and emission dispatch including valve point effect," in Proceedings of the 4th International Conference on Swarm, Evolutionary, and Memetic Computing, pp. 354-365, Springer, Chennai, India, December 2013.

[14] M. Ahmad, W. Ali, H. Farooq, M. Jamil, M. Ali, and A. U. Rehman, "Solving the problem of economic load dispatch for a small scale power system using a novel hybrid PSO-GSA algorithm," in Proceedings of the 2018 International Symposium on Recent Advances in Electrical Engineering (RAEE), pp. 1-6, IEEE, October 2018.

[15] M. A. El-hosseini, R. A. El-Sehiemy, and Y. H. Amira, "A multiobjective dynamic particle swarm optimizer for environmental/economic dispatch problem," Engineering Research Journal, vol. 37, no. 4, pp. 379-388, 2014.

[16] P. K. Hota, A. K. Barisal, and R. Chakrabarti, "Economic emission load dispatch through fuzzy based bacterial foraging algorithm," International Journal of Electrical Power and Energy Systems, vol. 32, no. 7, pp. 794-803, 2010.

[17] M. A. Abido, "A niched Pareto genetic algorithm for multiobjective environmental/economic dispatch," International 
Journal of Electrical Power \& Energy Systems, vol. 25, no. 2, pp. 97-105, 2003.

[18] S. Agrawal, B. K. Panigrahi, and M. K. Tiwari, "Multiobjective particle swarm algorithm with fuzzy clustering for electrical power dispatch," IEEE Trans. on Evolutionary Computation.vol. 12, no. 5, pp. 529-541, 2008.

[19] S. Reddy and K. Vaisakh, "Economic emission load dispatch by modified shuffled frog leaping algorithm," International Journal of Computer Applications, vol. 31, pp. 35-42, 2011.

[20] R. El-Sehiemy, M. A. El-Hosseini, and A. E. Hassanien, "Multiobjective real coded genetic algorithm for economic/ environmental dispatch problem," Studies in Informatics and Control, vol. 22, no. 2, pp. 113-122, 2013.

[21] K. Deb, Multi-objective Optimization Using Evolutionary Algorithms, Wiley, New York,NY, USA, 2001.

[22] C. M. Fonseca and P. J. Fleming, "An overview of evolutionary algorithms in multiobjective optimization," Evolutionary Computation, vol. 3, no. 1, pp. 1-16, 1995.

[23] A. A. Mousa and E. E. Elattar, "Best compromise alternative to EELD problem using hybrid multiobjective quantum genetic algorithm," International Journal of Applied Mathematics \& Information Sciences, vol. 8, no. 6, pp. 2889-2902, 2014.

[24] M. A. Mellal and E. J. Williams, "Cuckoo optimization algorithm with penalty function and binary approach for combined heat and power economic dispatch problem," Energy Reports, vol. 6, pp. 2720-2723, 2020.

[25] M. J. Kim, T. S. Kim, R. J. Flores, and J. Brouwer, "Neuralnetwork-based optimization for economic dispatch of combined heat and power systems," Applied Energy, vol. 265, Article ID 114785, 2020.

[26] A. Sundaram, "Multiobjective multi-verse optimization algorithm to solve combined economic, heat and power emission dispatch problems," Applied Soft Computing, vol. 91, Article ID 106195, 2020.

[27] M. F. Tabassum, M. Saeed, N. Ahmad Chaudhry et al., "Evolutionary simplex adaptive Hooke-Jeeves algorithm for economicload dispatch problem considering valve point loading effects," Ain Shams Engineering Journal, 2020.

[28] I. R. Fitri and J.-S. Kim, "Economic dispatch problem using load shedding: centralized solution," IFAC-Papers OnLine, vol. 52, no. 4, pp. 40-44, 2019.

[29] J. N. Kuk, R. A. Gonçalve, L. M. Pavelski, S. M. Guse Scós Venske, C. P. de Almeida, and A. T. Ramirez Pozo, "An empirical analysis of constraint handling on evolutionary multi-objective algorithms for the environmental/economic load dispatch problem," Expert Systems with Applications, vol. 165, Article ID 113774, 2021.

[30] D. Singh and J. S. Dhillon, "Ameliorated grey wolf optimization for economic load dispatch problem," Energy, vol. 169, pp. 398-419, 2019.

[31] O. T. Altinoz, "The distributed many-objective economic/ emission load dispatch benchmark problem," Swarm and Evolutionary Computation, vol. 49, pp. 102-113, 2019.

[32] A. Sundaram and P. Erdogmus, "Solution of combined economic emission dispatch problem with valve-point effect using hybrid NSGA II-MOPSO," in Particle Swarm Optimization with ApplicationsInTech, London, UK, 2017.

[33] X. Chen, K. Li, B. Xu, and Z. Yang, "Biogeography-based learning particle swarm optimization for combined heat and power economic dispatch problem," Knowledge-Based Systems, vol. 208, Article ID 106463, 2020.

[34] E. H. Talbi, L. Abaali, R. Skouri, and M. E. Moudden, "Solution of economic and environmental power dispatch problem of an electrical power system using BFGS-AL algorithm," Procedia Computer Science, vol. 170, pp. 857-862, 2020.

[35] S. Zhou, Z. Hu, W. Gu et al., "Combined heat and power system intelligent economic dispatch: a deep reinforcement learning approach," International Journal of Electrical Power \& Energy Systems, vol. 120, Article ID 106016, 2020.

[36] A. Srivastava and D. K. Das, "A new Kho-Kho optimization Algorithm: An application to solve combined emission economic dispatch and combined heat and power economic dispatch problem," Engineering Applications of Artificial Intelligence, vol. 94, Article ID 103763, 2020.

[37] R. Zhang, K. Yan, G. Li, T. Jiang, X. Li, and H. Chen, "Privacypreserving decentralized power system economic dispatch considering carbon capture power plants and carbon emission trading scheme via over-relaxed ADMM," International Journal of Electrical Power \& Energy Systems, vol. 121, Article ID 106094, 2020.

[38] C. A. C. Coello and A. Carlos, "A survey of constraint handling techniques used with evolutionary algorithms," Technical Report Lania-RI-99-04, Laboratorio Nacional de Informática Avanzada, Xalapa, Mexico, 1999.

[39] Z. Michalewicz, "A survey of constraint handling techniques in evolutionary computation methods," in Proceedings of the 4th Annual Conference on Evolutionary Programming, vol. 4, pp. 135-155, San Diego, CA, USA, February 1995.

[40] Q. Long, "A constraint handling technique for constrained multi-objective genetic algorithm," Swarm and Evolutionary Computation, vol. 15, pp. 66-79, 2014.

[41] Y. Yang, J. Liu, and S. Tan, "A constrained multi-objective evolutionary algorithm based on decomposition and dynamic constraint-handling mechanism," Applied Soft Computing, vol. 89, Article ID 106104, 2020.

[42] F. Samanipour and J. Jelovica, "Adaptive repair method for constraint handling in multi-objective genetic algorithm based on relationship between constraints and variables," Applied Soft Computing, vol. 90, Article ID 106143, 2020.

[43] J. Xu, P. Yang, G. Liu, Z. Bai, and W. Li, "Constraint handling in constrained optimization of a storage ring multi-bendachromat lattice," Nuclear Instruments and Methods in Physics Research Section A: Accelerators, Spectrometers, Detectors and Associated Equipment, vol. 988, Article ID 164890, 2020.

[44] A. A. Mousa and M. A. El-Shorbagy, "Identifying a satisfactory operation point for fuzzy multiobjective environmental/economic dispatch problem," American Journal of Mathematical and Computer Modelling, vol. 1, no. 1, pp. 1-14, 2016.

[45] A. A. Mousa, M. A. El-Shorbagy, and M. A. Farag, "K-meansClustering based evolutionary algorithm for multi-objective resource allocation problems," Applied Mathematics \& Information Sciences, vol. 11, no. 6, pp. 1-12, 2017.

[46] M. A. El-Shorbagy, A. Y. Ayoub, I. M. El-Desoky, and A. A. Mousa, "A novel genetic algorithm based k-means algorithm for cluster analysis," in International Conference on Advanced Machine Learning Technologies and Applications, pp. 92-101, Springer, Cham, Switzerland, 2018.

[47] H. P. Benson and S. Sayin, "Towards finding global representations of the efficient set in multiple objective mathematical programming," Naval Research Logistics, vol. 44, no. 1, pp. 47-67, 1997.

[48] A. K. Jain and R. C. Dubes, Algorithms for Clustering Data, Prentice-Hall, Englewood Cliffs, NJ, USA, 1988.

[49] M. A. E. Shorbagy, A. A. Mousa, and M. Farag, "Solving nonlinear single-unit commitment problem by genetic 
algorithm based clustering technique," Review of Computer Engineering Research, vol. 4, no. 1, pp. 11-29, 2017.

[50] P. Saurabh and B. Verma, "An efficient proactive artificial immune system based anomaly detection and prevention system," Expert Systems with Applications, vol. 60, pp. 311320, 2016.

[51] A. A. A. Mousa and M. A. El-Shorbagy, "Enhanced particle swarm optimization based local search for reactive power compensation problem," Applied Mathematics, vol. 3, no. 10, pp. 1276-1284, 2012.

[52] A. A. Mousa and I. M. El_Desoky, "Stability of Pareto optimal allocation of land reclamation by multistage decision-based multipheromone ant colony optimization," Swarm and Evolutionary Computation, vol. 13, pp. 13-21, 2013.

[53] D. Karaboga, "An idea based on honey bee swarm for numerical optimization," Technical Report-TR06, Erciyes University, Melikgazi, Turkey, 2005.

[54] W. Zhao and L. Wang, "An effective bacterial foraging optimizer for global optimization," Information Sciences, vol. 329, pp. 719-735, 2016.

[55] L. Guo, Z. Meng, Y. Sun, and L. Wang, "Parameter identification and sensitivity analysis of solar cell models with cat swarm optimization algorithm," Energy Conversion and Management, vol. 108, no. 15, pp. 520-528, 2016.

[56] M. Marinaki and Y. Marinakis, "A glowworm swarm optimization algorithm for the vehicle routing problem with stochastic demands," Expert Systems with Applications, vol. 46, pp. 145-163, 2016.

[57] S. Verma and V. Mukherjee, "Firefly algorithm for congestion management in deregulated environment," Engineering Science and Technology, An International Journal, vol. 19, no. 3, pp. 1254-1265, 2016.

[58] Y. Zhou, X. Chen, and G. Zhou, "An improved monkey algorithm for a 0-1 knapsack problem," Applied Soft Computing, vol. 38, pp. 817-830, 2016.

[59] A. L. A. Bolaji, M. A. Al-Betar, M. A. Awadallah, A. T. Khader, and L. M. Abualigah, "A comprehensive review: krill Herd algorithm (KH) and its applications," Applied Soft Computing, vol. 49, pp. 437-446, (2016).

[60] M. Shehab, A. T. Khader, M. Laouchedi, and O. A. Alomari, "Hybridizing cuckoo search algorithm with bat algorithm for global numerical optimization," The Journal of Supercomputing, vol. 75, no. 5, pp. 2395-2422, 2019.

[61] S. Mirjalili and A. Lewis, "The whale optimization algorithm," Advances in Engineering Software, vol. 95, pp. 51-67, 2016.

[62] Y. Abo-elnaga and M. A. El-Shorbagy, "Multi-sine cosine algorithm for solving nonlinear bilevel programming problems," International Journal of Computational Intelligence Systems, vol. 13, no. 1, pp. 421-432, 2020.

[63] M. A. El-Shorbagy and A. M. El-Refaey, "Hybridization of grasshopper optimization algorithm with genetic algorithm for solving system of non-linear equations," IEEE Access, vol. 8, pp. 220944-220961, 2020.

[64] S. Mirjalili, A. H. Gandomi, S. Z. Mirjalili, S. Saremi, H. Faris, and S. M. Mirjalili, "Salp Swarm Algorithm: a bio-inspired optimizer for engineering design problems," Advances in Engineering Software, vol. 114, pp. 163-191, 2017.

[65] A. Faramarzi, M. Heidarinejad, B. Stephens, and S. Mirjalili, "Equilibrium optimizer: a novel optimization algorithm," Knowledge-Based Systems, vol. 191, Article ID 105190, 2020.

[66] I. Ahmadianfar, O. Bozorg-Haddad, and X. Chu, "Gradientbased optimizer: a new Metaheuristic optimization algorithm," Information Sciences, Information Sciences, vol. 540, , pp. 131-159, 2020.
[67] S. Li, H. Chen, M. Wang, A. A. Heidari, and S. Mirjalili, "Slime mould algorithm: a new method for stochastic optimization," Future Generation Computer Systems, vol. 111, pp. 300-323, 2020.

[68] A. A. Heidari, S. Mirjalili, H. Faris, I. Aljarah, M. Mafarja, and H. Chen, "Harris hawks optimization: algorithm and applications," Future Generation Computer Systems, vol. 97, pp. 849-872, 2019.

[69] C. A. Coello Coello, S. González Brambila, J. Figueroa Gamboa et al., "Evolutionary multiobjective optimization: open research areas and some challenges lying ahead," Complex \& Intelligent Systems, vol. 6, pp. 221-236, 2020.

[70] A. A. Mousa, M. A. El-Shorbagy, and W. F. Abd El-Wahed, "Local search based hybrid particle swarm optimization for multiobjective optimization," International Journal of Swarm and Evolutionary Computation, vol. 3, pp. 1-14, 2012.

[71] K. Miettinen, Nonlinear Multiobjective Optimization, Kluwer Academic Publishers, Boston, MA, USA, 1999.

[72] J. Zahavi and L. Eisenberg, "Economic-environmental power dispatch," IEEE Transactions on Systems, Man, and Cybernetics, vol. 5, no. 5, pp. 485-489, 1985.

[73] D. Hazarika and P. K. Bordoloi, "Modified loss coefficients in the determination of optimum generation scheduling," IEE Proceedings C Generation, Transmission and Distribution, vol. 138, no. 2, pp. 166-172, 1991.

[74] W. Ng, "Generalized generation distribution factors for power system security evaluations," IEEE Transactions on Power Apparatus and Systems, vol. 100, no. 3, pp. 1001-1005, 1981.

[75] M. A. Farag, M. A. El-Shorbagy, A. A. Mousa, and I. M. ElDesoky, "A new hybrid metaheuristic algorithm for multiobjective optimization problems," International Journal of Computational Intelligence Systems-Atlantis Press Publisher, vol. 13, no. 1, pp. 920-940, 2020.

[76] M. A. El-Shorbagy, A. Y. Ayoub, A. A. Mousa, and I. M. ElDesoky, "An enhanced genetic algorithm with new mutation for cluster analysis," Computational Statistics, vol. 34, pp. 1355-1392, 2019.

[77] M. A. E. Shorbagy and A. A. Mousa, "Chaotic particle swarm optimization for imprecise combined economic and emission dispatch problem," Review of Information Engineering and Applications, vol. 4, no. 1, pp. 20-35, 2017.

[78] S. Hemamalini and P. S. Sishaj, "Emission constrained economic dispatch with valve point effect using particle swarm optimization," in Proceedings of the 2008 TENCON, Hyderabad, India, November 2008.

[79] R. Zimmerman and D. Gan, "MATPOWER: a matlab power system simulation package," 1997, http://www.pserc.cornell. edu/matpower/. 